\title{
Doping of Single Polymeric Nanoparticles
}

\author{
X-F Zhou, W Cheng and R G. Compton*
}

\begin{abstract}
The oxidative doping of single poly (Nvinylcarbazole) nanoparticles is reported in aqueous sodium perchlorate using nano-impacts. Complete oxidative doping of single PVK nanoparticles of size ca. $120 \mathrm{~nm}$ is demonstrated so validating for the first time a simple strategy to synthesise and characterise doped polymeric nanoparticles at the single nanoparticle level.
\end{abstract}

Polymeric nanoparticles are widely used in energy storage and other applications ${ }^{[1]}$. Charge transfer reactions using these materials involve the uptake or release of ions from the nanoparticulate polymer matrix and are generally studied by using an ensemble of nanoparticles in the form of a film or composite usually supported on an electrode surface ${ }^{[2]}$. For optimal performance, it is usually desirable that the nanoparticles can become fully charged / discharged. However, the unambiguous study of such processes is challenging when using an ensemble because the charge transfer is strongly coupled to mass transport processes which are typically impossible to quantify other than very approximately.

In this paper we show how the charging and doping of single polymeric nanoparticles can be studied for the first time using the newly developed method of nanoimpacts $^{[3]}$. In this method, a suspension of

[*] Mr. X.-F Zhou, Dr.W Cheng, Prof. Dr. R. G. Compton

Department of Chemistry

Physical \& Theoretical Chemistry Laboratory

Oxford University, South Parks Road, Oxford, OX1 3QZ, United Kingdom

Fax: (+44) 1865-275-410

Corresponding email: richard.compton@chem.ox.ac.uk nanoparticles is studied in situ using a microelectrode. The nanoparticles move randomly in solution with a Brownian motion which from time to time brings individual, single nanoparticles in contact with the electrode. If the latter is held at a suitable potential, the nanoparticle can be oxidatively (or reductively) doped and the event detected via a "spike" in a current-time transient.

Hitherto, the nano-impacts method has been applied to size nanoparticles ${ }^{[3 b, 3 e, 4]}$. In these experiments, charge transfer leads to dissolution of the nanoparticles, for example, $\mathrm{Ag}$ nanoparticles are oxidized to $\mathrm{Ag}^{+}$ions ${ }^{[3 b]}$, or indigo particles reduced to water soluble leucoindigo $^{[3 e]}$. Alternatively, mediated electron transfer can take place on the surface of impacted nanoparticles ${ }^{[5]}$ Namely, in the present paper, the oxidative doping of poly $(\mathrm{N}$-vinylcarbazole) nanoparticles is reported in aqueous sodium perchlorate using the nano-impacts method. It will be shown that the nanoparticles of ca. $120 \mathrm{~nm}$ diameter are essentially quantitatively oxidatively doped with the uptake of one perchlorate ion per carbazole monomer unit. PVK nanoparticles are important for electroluminescent devices [6] whilst the doping of PVK films is important in battery technology ${ }^{[7]}$.

First, to find the approximate potentials required for nano-impact experiments, cyclic voltammetry (CV) was used to study an ensemble of PVK NPs by modifying a macro glassy carbon (GC) electrode (diameter of $3 \mathrm{~mm}$ ) with different loadings of PVK 
nanoparticles (from $5 \times 10^{-11} \mathrm{~mol}$ up to $4 \times 10^{-10} \mathrm{~mol}$ of carbazole moieties applied to the electrode). Full experimental details appear in Supporting

Information. The oxidation potential for anion-doping of the PVK NPs in $1.0 \mathrm{M} \mathrm{NaClO}_{4}$ solution was determined to be ca. $+1.25 \mathrm{~V}$ (see FigureS1 in SP information). No peak was observed in backward scan or the second scan of $\mathrm{CV}$, indicating the oxidative doping of PVK nanoparticles is chemically irreversible and fully completed in $1.0 \mathrm{M} \mathrm{NaClO}_{4}$. Further, the number of electrons transferred per PVK monomer during the doping of PVK NPs was determined to be 2 \pm 0.1 by integrating the area of oxidative peak, namely the passed charge for oxidative doping versus number of monomer carbazole units (see FigureS2 in SP information). This suggests that the oxidative doping of PVK nanoparticles requires a two electron transfer process, which is consistent with literature reports ${ }^{[8]}$.

Next, a clean, unmodified carbon microelectrode was placed in $1.0 \mathrm{M} \mathrm{NaClO}_{4}$ solution and a known concentration of dispersed PVK nanoparticles added. +1.35 V ( vs. Saturated Calomel Electrode (SCE)) was used as the oxidative potential for the subsequent impact experiments to ensure that complete oxidation of PVK nanoparticles, as discussed above (see FigureS1 in SP information). Under potentiostatted conditions, at $+1.35 \mathrm{~V}$ vs. SCE, clear oxidative spikes from individual PVK nanoparticles were observed (Figure 1). The onset of these Faradaic spikes was found to be dependent on potential and no spike was observed at lower oxidation potentials such as $+0.4 \mathrm{~V}$ and $+0.6 \mathrm{~V}$, showing those spikes result from the oxidative doping of PVK NPs. A control experiment was conducted at the potential of $+1.35 \mathrm{~V}$ vs. SCE with no PVK nanoparticles in the solution, and no spikes were seen, confirming that the individual spikes is due to the random collisions of single PVK nanoparticles with the surface of the electrode and subsequent oxidative doping and electron transfer from the electrode.

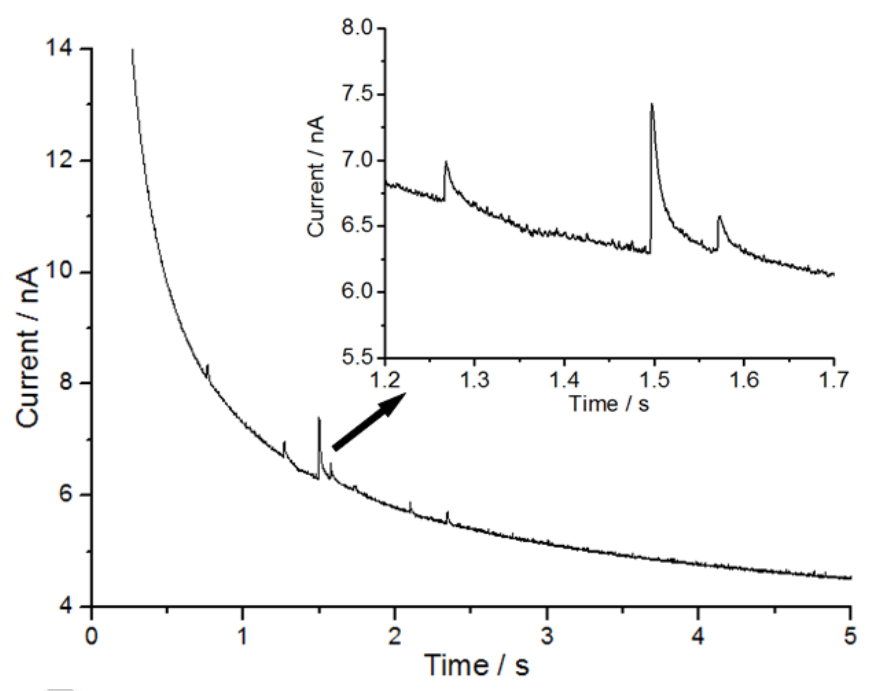

Figure 1 Typical chronoamperometric profiles showing oxidative Faradaic spikes of PVK nanoparticles in $1.0 \mathrm{M}$ $\mathrm{NaClO}_{4}$ at $+1.35 \mathrm{~V}$ vs. SCE. The inset shows the detailed impact spikes.

A total of 249 spikes were observed form 131 chronoamperograms of $5 \mathrm{~s}$ duration recorded at +1.35 $\mathrm{V}$, corresponding to the oxidative doping of 249 individual PVK nanoparticles. The charge of electrons during doping of single nanoparticles was then calculated by integrating the individual spikes area (see Figure S3 in SP information). This is the first time oxidative doping of single polymeric nanoparticles has been observed or measured.

To characterise the yield of oxidative doping of single polymeric nanoparticles, the modal size $D_{n p}$ (size distribution) of the same batch of PVK nanoparticles was estimated to be $121 \mathrm{~nm}$ through dynamic light 
scattering (DLS) (see FigureS3 in the SP information). If the charge is passed as a result of complete doping of single nanoparticles, then the theoretical charge $(Q)$ of electron transfer from complete doping of single polymeric nanoparticles can be determined by Equation 1, assuming that PVK nanoparticles are spherical.

$$
Q=\frac{D_{n p^{3} n F \pi \rho}}{6 M}
$$

Equation 1 where $\mathrm{D}_{n p}$ is the diameter of PVK nanoparticles, $\mathrm{M}$ is the molar mass of each PVK monomeric unit, $\rho$ is the density of PVK and F is the Faraday constant. The parameter $\mathrm{n}$ is the number of electrons transferred per PVK monomer during nanoparticles oxidation. The value of $n(n=2)$ was obtained from FigureS4 (See the SP information), as discussed above. Finally, by comparing the modal charge resulted from oxidative doping of 249 single polymeric nanoparticles $Q y(Q y=$ $1.23 \times 10^{-12} \mathrm{C}$ ) from experimental nano-impact method with the theoretical estimated maximum charge $Q(Q=$ $1.09 \times 10^{-12} \mathrm{C}$ ) of single polymeric nanoparticles, which indicates that there is effectively complete oxidative $(110 \% \pm 20 \%)$ doping of single polymeric PVK nanoparticles. The slightly larger estimated charge may be attributed to the aggregation/ agglomeration of polymeric nanoparticles in high concentration of sodium perchloride medium during the course of the nano-impact experiments.

In addition, there was a good agreement with the distribution of charge from the oxidative doping by nano-impact method, as analysed from the above equation for all 249 single polymeric PVK nanoparticle and the theoretical estimation (Figure 2).
The similar normal distribution (see SP information for details) further indicates complete oxidative doping of individual polymeric PVK nanoparticles even if there is size distribution of the analysed individual nanoparticles. The results show first almost complete oxidative doping of single polymeric PVK nanoparticles and quantitative analysis of doping yield of single polymeric NPs is viable using the nanoimpact method. Second it shows that the PVK nanoparticles are quantitatively oxidized with the uptake of one perchlorate ion per carbazole monomer unit, as illustrated in Scheme 1, based on the expected electrooxidation of $\mathrm{PVK}^{[8]}$.

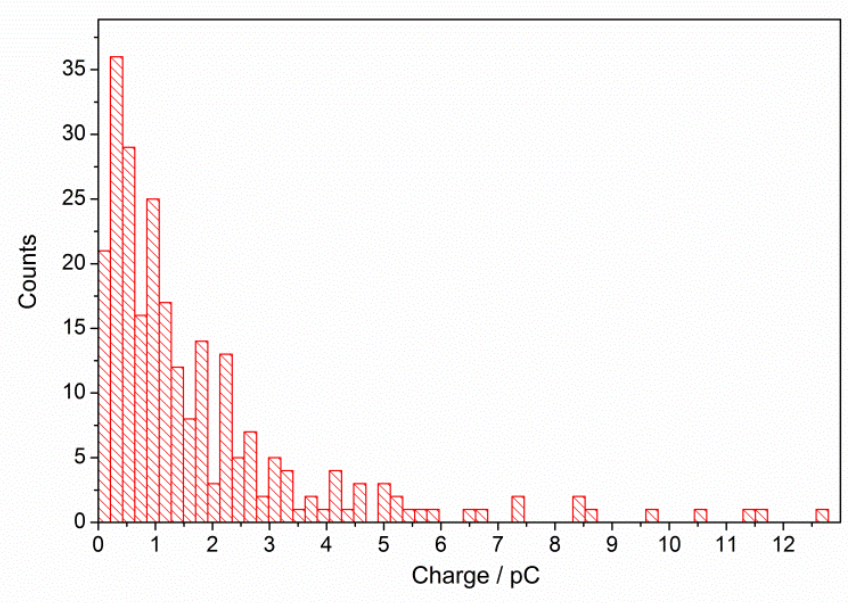

(a)

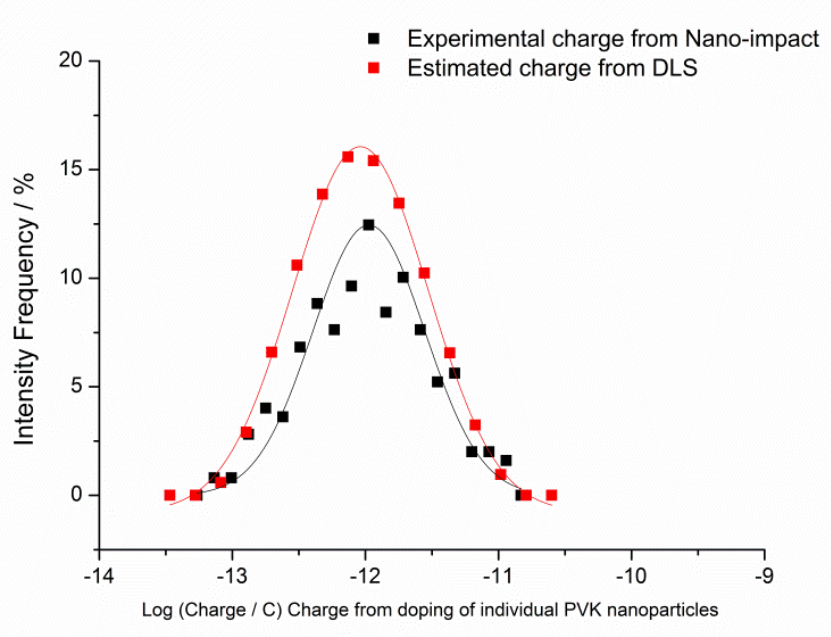

(b) 
Figure 2 (a) shows the distribution of charge from oxidative doping of single PVK nanoparticles by analysis of the 249 oxidation spikes from nano-impact experiments. Histogram shows the charge distribution of single PVK nanoparticles derived from charge per spike from nanoimpact experiments and the theoretical estimated from DLS data (see SP information) according to Equation 1.

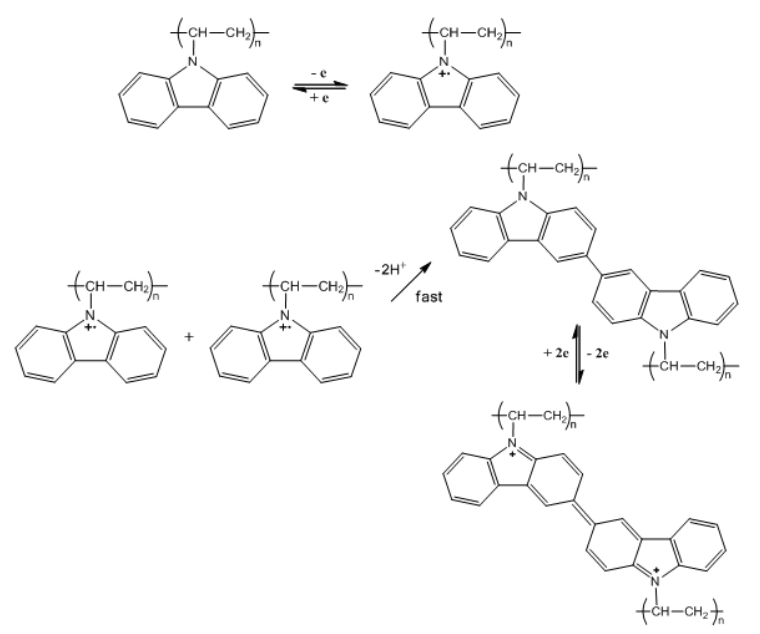

Scheme 1 Suggested mechanism of PVK nanoparticle oxidation based on [8].

To our best of knowledge, no studies have been reported to demonstrate doping of single individual polymeric nanoparticles. In this communication, we have shown the nano-impacts for doping single polymeric nanoparticles and further demonstrated how to characterise the doping yield from doped single nanoparticles. We believe this strategy will have major application in both synthesis and characterisation of doped polymeric nanoparticles for new materials and energy applications, in addition, providing unique characterisation of polymeric single nanoparticles such as dopant effect and doping yield which are difficult to characterise by other spectroscopic and electron microscopic methods.

\section{Acknowledgements}

The European Research Council under the European Union's Seventh Framework Programme (ERC Grant Agreement n. [320403]) is gratefully acknowledged for partly funding this work. We thank Dr. D Omanović (Center for Marine and Environmental Research Zagreb, Croatia) for developing Signal Counter software for data analysis, and the Leverhulme Trust for support for WC.

Keywords: Polymeric nanoparticle • Electrochemistry • Doping - Nano-impacts

[1] a) Q. F. Zhang, E. Uchaker, S. L. Candelaria, G. Z. Cao, Chem Soc Rev 2013, 42, 3127-3171; b) A. R. Kirtane, J. Panyam, Nat Nanotechnol 2013, 8, 805806.c) K. Y. Pu, A. J. Shuhendler, J. V. Jokerst, J. G. Mei, S. S. Gambhir, Z. N. Bao, J. H. Rao, Nat Nanotechnol 2014, 9, 233-239; d) F. H. Schacher, P. A. Rupar, I. Manners, Angew Chem Int Edit 2012, 51, 7898-7921.e) Y. L. Colson, M. W. Grinstaff, Advanced Materials 2012, 24, 3878-3886; f) K. Jaskiewicz, A. Larsen, I. Lieberwirth, K. Koynov, W. Meier, G. Fytas, A. Kroeger, K. Landfester, Angew Chem Int Edit 2012, 51, 4613-4617.

[2] a) A. G. MacDiarmid, Angew Chem Int Edit 2001, 40, 2581-2590; b) C. Li, H. Bai, G. Q. Shi, Chem Soc Rev 2009, 38, 23972409.

[3] a) S. E. Kleijn, S. C. Lai, M. T. Koper, P. R. Unwin, Angew Chem Int Edit 2014, 53, 3558-3586; b) Y. G. Zhou, N. V. Rees, R. G. Compton, Angew Chem Int Edit 2011, 
50, 4219-4221; c) X. Y. Xiao, F. R. F. Fan, J. P. Zhou, A. J. Bard, J Am Chem Soc 2008, 130, 16669-16677; d) X. Y. Xiao, A.

J. Bard, J Am Chem Soc 2007, 129, 96109612; e) W. Cheng, X.-F. Zhou, R. G. Compton, Angew. Chem. Int. Ed. 2013, 52, 12980-12982; f) B. K. Kim, A. Boika, J. Kim, J. E. Dick, A. J. Bard, J Am Chem Soc 2014, 136, 4849-4852; g) W. Cheng, R. G. Compton, Trends in Analytical Chemistry 2014, 58, 79-89; h) A. J. Bard, H. J. Zhou, S. J. Kwon, Isr J Chem 2010 , 50, 267-276.

a) Y. G. Zhou, N. V. Rees, J. Pillay, R. Tshikhudo, S. Vilakazi, R. G. Compton, Chem Commun 2012, 48, 224-226; b) K. Tschulik, B. Haddou, D. Omanović, N. V. Rees, R. G. Compton, Nano Research 2013, 6, 836-841.

[5] a) S. E. F. Kleijn, S. C. S. Lai, T. S. Miller, A. I. Yanson, M. T. M. Koper, P. R. Unwin, J Am Chem Soc 2012, 134, 1855818561; b) J. H. Park, S. N. Thorgaard, B. Zhang, A. J. Bard, J Am Chem Soc 2013, 135, 5258-5261; c) D. Wakerley, A. G. Guell, L. A. Hutton, T. S. Miller, A. J. Bard, J. V. Macpherson, Chem Commun (Camb) 2013, 49, 5657-5659; d) R. Dasari, D. A. Robinson, K. J. Stevenson, J Am Chem Soc 2013, 135, 570-573; e) S. E.
Fosdick, M. J. Anderson, E. G. Nettleton, R. M. Crooks, J Am Chem Soc 2013, 135, 5994-5997; f) J. M. Kahk, N. V. Rees, J. Pillay, R. Tshikhudo, S. Vilakazi, R. G. Compton, Nano Today 2012, 7, 174-179.

a) C. F. Huebner, J. B. Carroll, D. D.

Evanoff, Y. R. Ying, B. J. Stevenson, J. R. Lawrence, J. M. Houchins, A. L. Foguth, J. Sperry, S. H. Foulger, J Mater Chem 2008, 18, 4942-4948; b) C. Martin, S.

Bhattacharyya, A. Patra, A. Douhal, Photochemical \& Photobiological Sciences 2014, DOI: 10.1039/c4pp00086b c) C. Y. Jiang, W. Yang, J. B. Peng, S. Xiao, Y. Cao, Adv Mater 2004, 16, 537541.

[7] a) F. C. Chen, Y. Yang, M. E. Thompson, J. Kido, Appl Phys Lett 2002, 80, 23082310; b) J. Jang, Y. Nam, H. Yoon, $A d v$ Mater 2005, 17, 1382-1386.

[8] a) R. G. Compton, F. J. Davis, S. C. Grant, J Appl Electrochem 1986, 16, 239-249; b) S.N. Bhadani, J Appl Polym Sci 1991, 42, 1271-1273; c) A. Desbenemonvernay, J. E. Dubois, P. C. Lacaze, J Electroanal Chem 1985, 189, 51-63. 


\section{COMMUNICATION}

Oxidative doping and quantitative analysis of doping yield of single polymeric NPs is demonstrated. This provides a simple unique strategy to synthesise and characterise doped polymeric nanoparticles at single NP level.

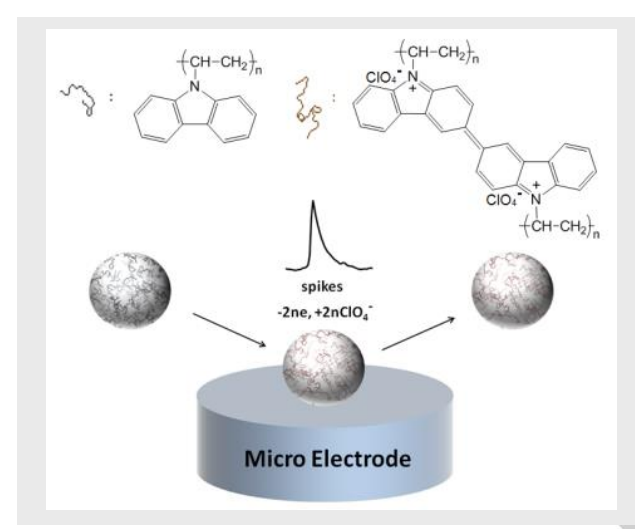

X-F Zhou, W Cheng and R G. Compton*

Page 1. - Page 6.

Doping of Single Polymeric Nanoparticles 